\title{
LONG MEMORY AT THE LONG-RUN AND THE SEASONAL MONTHLY FREQUENCIES IN THE US MONEY STOCK
}

\author{
Guglielmo Maria Caporale
}

Brunel University, London

\author{
Luis A. Gil-Alana
}

University of Navarra

\begin{abstract}
In this paper we show that the monthly structure of the US money stock can be specified in terms of a long-memory process, with roots at both the zero and the seasonal monthly frequencies. We use a procedure that enables us to test simultaneously for the roots at all these frequencies. The results show that the root at the long-run or zero frequency plays a much more important role than the seasonal one, though the latter should also be taken into account.
\end{abstract}

Keywords: $\quad$ Seasonality, Long Memory, Fractional Integration

JEL Classification: C15; C22

Corresponding author: Professor Guglielmo Maria Caporale, Brunel Business School, Brunel University, Uxbridge, Middlesex UB8 3PH, UK. Tel.: +44 (0)1895 266713. Fax: +44 (0)1895 269770. Email: Guglielmo-Maria.Caporale@brunel.ac.uk

The second named author gratefully acknowledges financial support from the PIUNA Project at the University of Navarra, Pamplona, Spain. 


\section{Introduction}

Dickey, Hasza and Fuller (DHF, 1984), Hylleberg, Engle, Granger and Yoo (HEGY, 1990), Beaulieu and Miron (1993), and Tam and Reinsel (1998), amongst others, have proposed test statistics for seasonal unit roots in raw time series. More precisely, if $x_{t}$ is the time series we observe, with a changing seasonal pattern, we can consider the model

$$
\left(1-L^{s}\right) x_{t}=u_{t}, t=1,2, \ldots
$$

where $s$ is the number of time periods in a year, and $u_{t}$ is an $I(0)$ process, defined for the purposes of the present paper as a covariance-stationary process with spectral density that is positive and finite at any frequency. Note that the polynomial in (1) can be decomposed into $(1-L)\left(1+L+L^{2}+\ldots+L^{s-1}\right)=(1-L) S(L)$. That is, the seasonal difference operator can be written as the product of the first difference operator and the moving-average filter $\mathrm{S}(\mathrm{L})$, containing further roots of modulus unity. The root at the long-run or zero frequency then appears as a component of the seasonal polynomial in (1). However, there are many cases when this frequency plays a major role, accounting not only for some of the seasonal behaviour but also for the trending stochastic behaviour of the series.

In this paper, we focus on monthly data, (i.e. $\mathrm{s}=12$ ), and present a version of the testing procedure of Robinson (1994) that enables us to consider simultaneously unit roots with possibly fractional orders of integration at both the zero and the seasonal frequencies. In particular, we examine models such as:

$$
(1-L)^{d_{1}}\left(1-L^{12}\right)^{d_{2}} x_{t}=u_{t}, \quad t=1,2, \ldots,
$$

for given real values $d_{1}$ and $d_{2}$. Here, the first fractional polynomial can be expressed in terms of its binomial expansion such that, for all real values $d_{1}$,

$$
(1-L)^{d_{1}}=\sum_{j=0}^{\infty}\left(\begin{array}{l}
d_{1} \\
j
\end{array}\right)(-1)^{j} L^{j}=1-d_{1} L+\frac{d_{1}\left(d_{1}-1\right)}{2} L^{2}-\ldots
$$

and similarly, for the seasonal component, 


$$
\left(1-L^{12}\right)^{d_{2}}=\sum_{j=0}^{\infty}\left(\begin{array}{l}
d_{2} \\
j
\end{array}\right)(-1)^{j} L^{12 j}=1-d_{2} L^{12}+\frac{d_{2}\left(d_{2}-1\right)}{2} L^{24}-\ldots
$$

Clearly, setting $d_{1}=1$ and $d_{2}=0$ in (2) amounts to testing the classical unit root model (Dickey and Fuller, 1979; Phillips, 1987; etc); if $\mathrm{d}_{1}=0$ and $\mathrm{d}_{2}=1$, we have seasonal unit roots (e.g., HEGY, 1990), and if $\mathrm{d}_{1}=\mathrm{d}_{2}=1$, we obtain the "airline" model introduced by Box and Jenkins (1976).

\section{The testing procedure}

We use a simple version of the tests of Robinson (1994), specifically a Lagrange Multiplier (LM) test of the null hypothesis:

$$
H_{o}: d \equiv\left(d_{1}, d_{2}\right)^{\prime}=\left(d_{1 o}, d_{2 o}\right)^{\prime} \equiv d_{o},
$$

in (2) for given real numbers $d_{10}$ and $d_{20}$. The test statistic is given by:

$$
\hat{R}=\frac{T}{\hat{\sigma}^{4}} \hat{a}^{\prime} \hat{A}^{-1} \hat{a},
$$

where $\mathrm{T}$ is the sample size, and

$$
\begin{aligned}
& \hat{a}=\frac{-2 \pi}{T} \sum_{j}^{*} \psi\left(\lambda_{j}\right) g\left(\lambda_{j} ; \hat{\tau}\right)^{-1} I\left(\lambda_{j}\right) ; \quad \hat{\sigma}^{2}=\sigma^{2}(\hat{\tau})=\frac{2 \pi}{T} \sum_{j=1}^{T-1} g\left(\lambda_{j} ; \hat{\tau}\right)^{-1} I\left(\lambda_{j}\right), \\
& \hat{A}=\frac{2}{T}\left(\sum_{j}^{*} \psi\left(\lambda_{j}\right) \psi\left(\lambda_{j}\right)^{\prime}-\sum_{j}^{*} \psi\left(\lambda_{j}\right) \hat{\varepsilon}\left(\lambda_{j}\right)^{\prime}\left(\sum_{j}^{*} \hat{\varepsilon}\left(\lambda_{j}\right) \hat{\varepsilon}\left(\lambda_{j}\right)^{\prime}\right)^{-1} \sum_{j}^{*} \hat{\varepsilon}\left(\lambda_{j}\right) \psi\left(\lambda_{j}\right)^{\prime}\right) \\
& \psi\left(\lambda_{j}\right)^{\prime}=\left\lfloor\psi_{1}\left(\lambda_{j}\right), \psi_{2}\left(\lambda_{j}\right)\right\rfloor ; \quad \hat{\varepsilon}\left(\lambda_{j}\right)=\frac{\partial}{\partial \tau} \log g\left(\lambda_{j} ; \hat{\tau}\right) ; \quad \psi_{1}\left(\lambda_{j}\right)=\log \left|2 \sin \frac{\lambda_{j}}{2}\right| ; \\
& \psi_{2}\left(\lambda_{j}\right)=\log \left|2 \sin \frac{\lambda_{j}}{2}\right|+\log \left(2 \cos \frac{\lambda_{j}}{2}\right)+\log \left|2 \cos \lambda_{j}\right|+\log 2\left(\cos \lambda_{j}-\cos \frac{\pi}{3}\right) \mid+ \\
& +\log \left|2\left(\cos \lambda-\cos \frac{2 \pi}{3}\right)\right|+\log \left|2\left(\cos \lambda-\cos \frac{\pi}{6}\right)\right|+\log \left|2\left(\cos \lambda-\cos \frac{5 \pi}{6}\right)\right|,
\end{aligned}
$$


with $\lambda_{\mathrm{j}}=2 \pi \mathrm{j} / \mathrm{T}$. $\mathrm{I}\left(\lambda_{\mathrm{j}}\right)$ is the periodogram of $\hat{u}_{t}=(1-L)^{d_{10}}\left(1-L^{12}\right)^{d_{20}} x_{t}$, and $\hat{\tau}=$ $\arg \min _{\tau \in T^{*}} \sigma^{2}(\tau)$, with $\mathrm{T}^{*}$ as a suitable subset of the $\mathrm{R}^{\mathrm{q}}$ Euclidean space. Finally, the function $\mathrm{g}$ above is a known function coming from the spectral density of $\mathrm{u}_{\mathrm{t}}$ :

$$
f(\lambda ; \tau)=\frac{\sigma^{2}}{2 \pi} g(\lambda ; \tau), \quad-\pi<\lambda \leq \pi
$$

Note that these tests are purely parametric, and therefore require specific modelling assumptions about the short-memory specification of $\mathrm{u}_{\mathrm{t}}$. For instance, if $\mathrm{u}_{\mathrm{t}}$ is a white noise process, $g \equiv 1$, whilst if $\mathrm{u}_{\mathrm{t}}$ is an AR process of the form $\phi(\mathrm{L}) \mathrm{u}_{\mathrm{t}}=\varepsilon_{\mathrm{t}}$, then $\mathrm{g}=\left|\phi\left(\mathrm{e}^{\mathrm{i} \lambda}\right)\right|^{-2}$, with $\sigma^{2}=$ $\mathrm{V}\left(\varepsilon_{\mathrm{t}}\right)$, the AR coefficients being a function of $\tau$.

Based on $\mathrm{H}_{\mathrm{o}}$ (3), Robinson (1994) established that, under certain regularity conditions:

$$
\hat{R} \rightarrow_{d} \quad \chi_{2}^{2}, \quad \text { as } \quad T \rightarrow \infty
$$

Thus, unlike other procedures, we are in a classical large-sample testing situation for the reasons outlined by Robinson (1994), who also showed that the tests are efficient in the Pitman sense against local departures from the null. A test of (3) will reject $\mathrm{H}_{\mathrm{o}}$ against the alternative $\mathrm{H}_{\mathrm{a}}: \mathrm{d} \neq \mathrm{d}_{\mathrm{o}}$ if $\hat{R}>\chi_{2, \alpha}^{2}$, where Prob $\left(\chi_{2, \alpha}^{2}>\chi_{2}^{2}\right)=\alpha$. There exist other versions of the tests of Robinson (1994), testing, for example, only the root at the long-run or zero frequency (e.g., Gil-Alana and Robinson, 1997; Gil-Alana, 2000), purely seasonal fractional models (GilAlana, 1999, 2002; Gil-Alana and Robinson, 2001), or cyclical structures (Gil-Alana, 2001). However, a simultaneous test for the roots at both the zero and the seasonal monthly components has not been implemented yet. We carry out such a test in the following section.

\section{Testing for the orders of integration of the US monthly money stock}

The time series analysed in this section is the (seasonally unadjusted) US monthly money stock (billions of dollars), for the time period $1947 \mathrm{ml}$ to $2002 \mathrm{~m} 8$, obtained from the Federal Reserve Bank of St. Louis database. 
Denoting the time series $x_{t}$, we employ throughout model (2), testing $H_{o}$ (3) for values $\mathrm{d}_{1 \mathrm{o}}, \mathrm{d}_{2 \mathrm{o}}=0,(0.25), 2$, thereby including tests for a unit root exclusively at the long-run or zero frequency $\left(d_{1 o}=1, d_{2 o}=0\right)$, as well as tests for seasonal unit roots $\left(d_{1 o}=0, d_{2 o}=1\right)$, and unit and seasonal unit roots $\left(\mathrm{d}_{1 \mathrm{o}}=\mathrm{d}_{2 \mathrm{o}}=1\right)$, in addition to other fractionally integrated possibilities. Initially, we assume that $\mathrm{u}_{\mathrm{t}}$ is white noise, but then we also allow for weakly parametrically autocorrelated disturbances. In particular, we consider AR(1) and seasonally monthly AR(1) processes for $u_{t}$. Only one non-rejection occurs for the three types of disturbances, corresponding to $\left(\mathrm{d}_{10}, \mathrm{~d}_{20}\right)=(1.25,0.25)$. This suggests that the order of integration at the longrun frequency has a much more important role than the one corresponding to the seasonal frequency.

\section{(Insert Figure 1 about here)}

In order to have a more precise view about the non-rejection values, we performed again the tests of Robinson (1994), but this time using increments of 0.01 for $\mathrm{d}_{1 \mathrm{o}}$ and $\mathrm{d}_{2 \mathrm{o}}$. Figure 1 displays the non-rejection regions of $\left(\mathrm{d}_{10}, \mathrm{~d}_{20}\right)$ for each type of disturbances. It can be seen that in all cases $d_{10}$ is higher than $d_{20}$, highlighting once more the importance of the root at the zero frequency. In particular, $\mathrm{d}_{10}$ appears to be constrained between 1.1 and 1.5 , whilst $\mathrm{d}_{2 \mathrm{o}}$ oscillates around 0.25 . Consequently, shocks to the long-run component will have permanent effects, policy actions being required to bring the series back to its original trend. On the other hand, seasonal shocks will be transitory, mean reversion occurring at some point in the future.

Next, we try to establish what might be the best model specification for this series. For this purpose, we compute, for each model, the values of $d_{10}$ and $d_{20}$ producing the lowest statistics, which should be an approximation of the maximum likelihood estimates; this is because the procedure employed here is a Lagrange Multiplier test and is based on the Whittle function, which is an approximation to the likelihood function. The parameter values are displayed in Table 1. 
It can be seen that, if $\mathrm{u}_{\mathrm{t}}$ is white noise, $\mathrm{d}_{1}=1.17$ and $\mathrm{d}_{2}=0.14$; the corresponding values if $\mathrm{u}_{\mathrm{t}}$ is $\mathrm{AR}(1)$ are $\mathrm{d}_{1}=1.10$ and $\mathrm{d}_{2}=0.09$; finally, if $\mathrm{u}_{\mathrm{t}}$ is modelled as a seasonal AR(1) process, $\mathrm{d}_{1}=1.22$ and $\mathrm{d}_{2}=0.29$. Thus, in all three cases the order of integration at the zero frequency is higher than 1, whilst the seasonal one is slightly above 0 . Several diagnostic tests were then carried out on the residuals of the estimated models, indicating that the best model is the one with seasonal AR disturbances. Our preferred specification is therefore the following:

$$
\begin{gathered}
(1-L)^{1.22}\left(1-L^{12}\right)^{0.29} x_{t}=u_{t}, \quad t=1,2, \ldots \\
u_{t}=-0.143 u_{t-1}+\varepsilon_{t}, \quad t=1,2, \ldots
\end{gathered}
$$

with white noise $\varepsilon_{t}$. Clearly, the standard approach of taking first differences or first seasonal differences would not appropriate here, since the former would result in a series still exhibiting a long-memory component, whilst the latter would entail overdifferencing.

\section{Conclusions}

The stochastic behaviour of the US money stock has been examined in this paper using a procedure that enables us to consider simultaneously roots with fractional orders of integration both at the zero and the seasonal frequencies. The results suggest that the root at the zero frequency should be considered independently of the seasonal frequency, though the latter should also be taken into account, exhibiting an order of integration of about 0.25 . Finally, the fact that the root at the long-run frequency is higher than 1 , while the one affecting the seasonal structure is smaller than 1 , has some implications in terms of policy action and forecasting. In particular, whilst shocks affecting the seasonal structure appear to be mean reverting, those affecting the long run tend to persist forever, requiring policy-makers to take appropriate actions to restore equilibrium. 


\section{References}

Beaulieu, J.J. and J.A. Miron, 1993, Seasonal unit roots in aggregate U.S. data, Journal of Econometrics 55, 305-328.

Box, G.E.P. and G.M. Jenkins, 1976, Time Series Analysis: Forecasting and Control, $\left(2^{\text {nd }}\right.$ eds. $)$ San Francisco, C.A.: Holden-Day.

Dickey, D. A., D. P. Hasza and W. A. Fuller, 1984, Testing for unit roots in seasonal time series, Journal of the American Statistical Association 79, 355-367.

Gil-Alana, L.A., 1999, Testing fractional integration with monthly data, Economic Modelling $16,613-629$.

Gil-Alana, L.A., 2000, Mean reversion in the real exchange rates, Economics Letters 69, 285288.

Gil-Alana, L.A., 2001, Testing stochastic cycles in macroeconomic time series, Journal of Time Series Analysis 22, 411-430.

Gil-Alana, L.A., 2002, Seasonal long memory in the aggregate output, Economics Letters 74, 333-337.

Gil-Alana, L.A. and P.M. Robinson, 1997, Testing of unit roots and other nonstationary hypotheses in macroeconomic time series, Journal of Econometrics 80, 241-268.

Gil-Alana, L.A. and P.M. Robinson, 2001, Testing seasonal fractional integration in the UK and Japanese consumption and income, Journal of Applied Econometrics 16, 95-114.

Hylleberg, S., R.F. Engle, C.W.J. Granger and B.S. Yoo, 1990, Seasonal integration and cointegration, Journal of Econometrics 44, 215-238.

Porter-Hudak, S., 1990, An application of the seasonal fractionally differenced model to the monetary aggregates, Journal of the American Statistical Association 85, 338-344.

Robinson, P.M., 1994, Efficient tests of nonstationary hypotheses, Journal of the American Statistical Association 89, 1420-1437.

Tam, W. and G. C. Reimsel, 1997, Tests for seasonal moving average unit root in ARIMA models, Journal of the American Statistical Association 92, 725-738. 


\section{FIGURE 1}

Region of values of $d_{10}$ and $d_{20}$ where $H_{0}$ (3) cannot be rejected at the $95 \%$ significance level

i) with white noise disturbances:

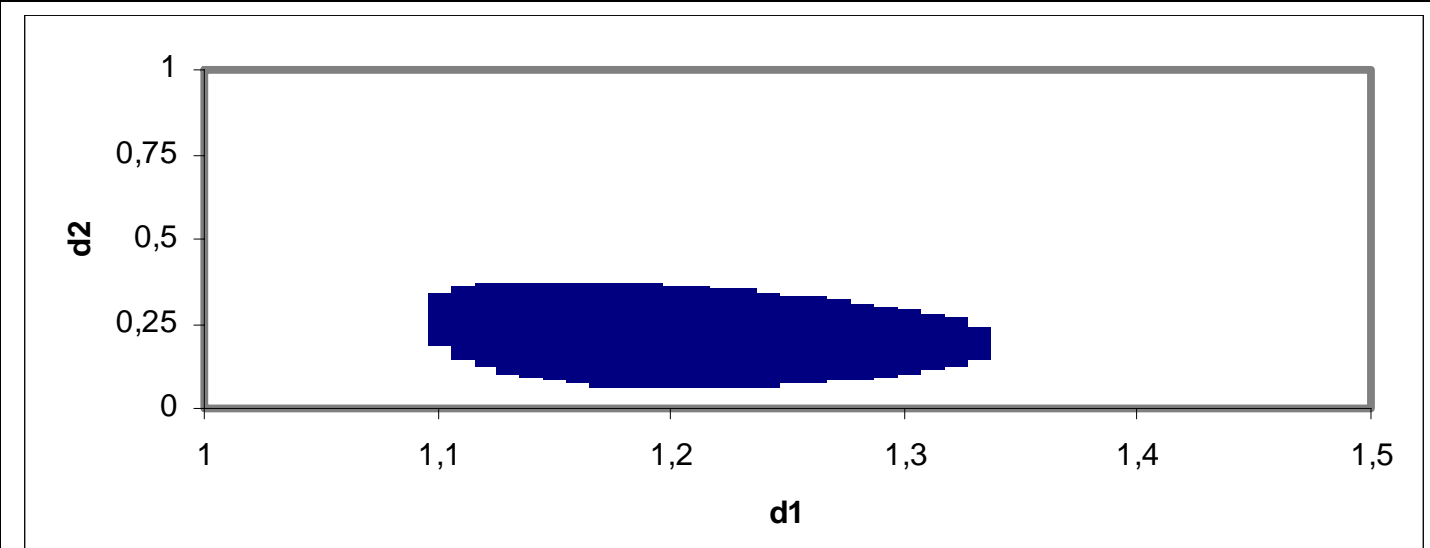

ii) with AR(1) disturbances:

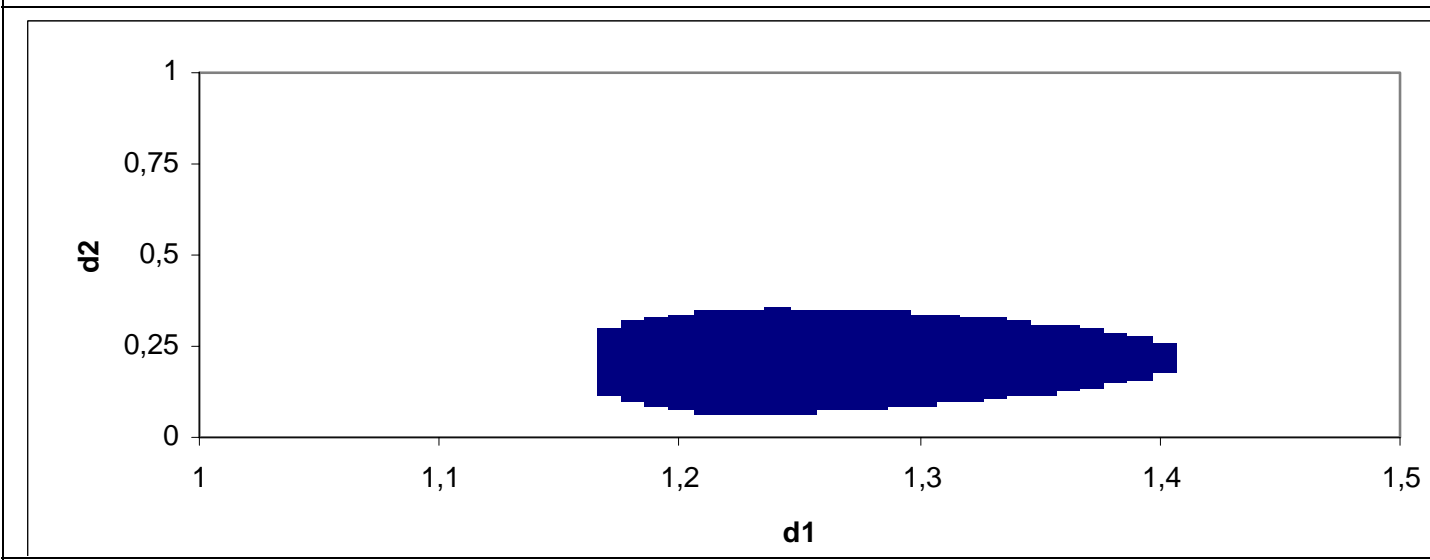

iii) with seasonal AR(1) disturbances:

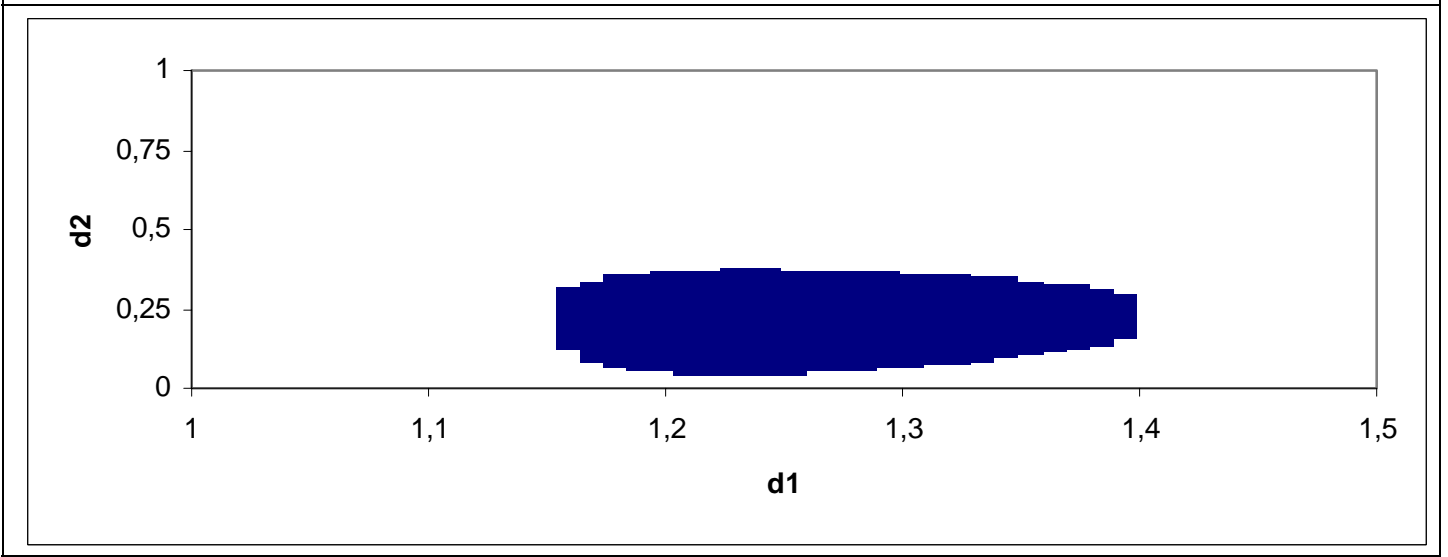




\section{TABLE 1}

Model specifications according to the lowest statistics in Figure 1

\begin{tabular}{|c|c|c|c|c|}
\hline $\mathrm{u}_{\mathrm{t}}$ & $\mathrm{d}_{1}$ & $\mathrm{~d}_{2}$ & AR coeff. & Seasonal AR coeff. \\
\hline White noise & 1.17 & 0.14 & ---- & ---- \\
\hline AR (1) & 1.10 & 0.09 & 0.042 & ---- \\
\hline Seasonal AR(1) & 1.22 & 0.29 & --- & -0.143 \\
\hline
\end{tabular}

